\title{
Reduced expression of sushi domain containing 2 is associated with progression of non-small cell lung cancer
}

\author{
CUIXIA CAI, RONG SHI, YUAN GAO, JUN ZENG, MIN WEI, \\ HANDUO WANG, WENLING ZHENG and WENLI MA \\ Institute of Genetic Engineering, Southern Medical University, Guangzhou, Guangdong 510515, P.R. China
}

Received October 30, 2014; Accepted August 25, 2015

DOI: $10.3892 / \mathrm{ol} .2015 .3737$

\begin{abstract}
Sushi domain containing 2 (SUSD2) has been identified as a gene encoding an 822-amino acid protein, which contains a transmembrane domain and functional domains inherent to adhesion molecules. Previous studies have reported that increased expression of SUSD2 has a critical role in tumorigenesis in human breast cancer. However, to the best of our knowledge, SUSD2 expression status and its correlation with the clinicopathological features of non-small cell lung cancer (NSCLC) have not previously been investigated. In the present study, reverse transcription-quantitative polymerase chain reaction and western blotting were used to evaluate SUSD2 messenger RNA (mRNA) and protein expression in NSCLC and adjacent normal lung tissues. The clinicopathological significance of SUSD2 was investigated by immunohistochemical analysis of an NSCLC tissue microarray. Receiver operating characteristic analysis was used to determine the cut-off score for positive expression of SUSD2. Furthermore, the correlation between SUSD2 expression and the clinicopathological features of NSCLC was analyzed by $\chi^{2}$ test. The results revealed that SUSD2 mRNA $(\mathrm{P}<0.0001)$ and protein $(\mathrm{P}<0.0001)$ expression levels were significantly decreased in NSCLC tissues compared with those of adjacent normal tissues. When the SUSD2 positive expression percentage was determined to be $>47.5 \%$ (area under ROC curve, 0.799; $\mathrm{P}<0.000$ ), positive expression of SUSD2 was observed in $100 \%(32 / 32)$ of normal lung tissues and 55\% (88/160) of NSCLC tissues by immunohistochemistry $\left(\chi^{2}=21.160 ; \mathrm{P}<0.000\right)$. Furthermore, it was demonstrated that the reduced SUSD2 protein levels in cancer tissues were positively correlated with poor histological grade $\left(\chi^{2}=41.764 ; \mathrm{P}<0.000\right)$, advanced clinical stage $\left(\chi^{2}=10.790 ; \mathrm{P}=0.013\right)$, higher $\mathrm{pT}\left(\chi^{2}=9.070 ; \mathrm{P}=0.028\right)$ and positive regional lymph node metastasis $\left(\chi^{2}=15.399 ; \mathrm{P}=0.002\right)$.
\end{abstract}

Correspondence to: $\mathrm{Dr}$ Wenli Ma, Institute of Genetic Engineering, Southern Medical University, 1838 North Guangzhou Avenue, Guangzhou, Guangdong 510515, P.R. China

E-mail:wenli668@gmail.com

Key words: sushi domain containing 2, non-small cell lung cancer, tissue microarray, immunohistochemistry
In conclusion, these data suggest that the reduced expression of SUSD2 is associated with the progression of NSCLC and may have a role in the pathogenesis of NSCLC.

\section{Introduction}

Lung cancer is the leading cause of cancer-associated mortalities worldwide (1). Non-small cell lung cancer (NSCLC), which includes adenocarcinoma, squamous cell carcinoma and large cell carcinoma, comprises $85 \%$ of lung cancer $(2,3)$. Despite improvements to the treatment of lung cancer, the overall 5 -year survival rate has remained poor at $<15 \%(3,4)$. Therefore, the search for effective biomarkers for early diagnosis, prognosis and individualized medication treatment plans for NSCLC has become a significant focus of recent research.

Sushi domain containing 2 (SUSD2) is located on chromosome 22 and encodes an 822-amino acid type I membrane protein, including somatomedin B, AMOP, von Willebrand factor type D and sushi domains, which have significant roles in the mediation of cell-cell and cell-matrix adhesion in molecules (5). Two studies by Sugahara et al $(6,7)$ investigated the mouse homolog: SUSD2 (also known as mSVS-1 or SVS-1) was found to be downregulated in activated oncogene-v-K-ras-transformed NIH3T3 cells (Ki3T3 cells), compared with mouse NIH3T3 cells. One of the studies (6) revealed that overexpression of SUSD2 in HT1080 fibrosarcoma cells and HeLa cervical carcinoma cells inhibited clonogenicity, anchorage-independent growth, migration and invasion, via Matrigel assays. Simultaneously, Sugahara et al (7) also suggested that SUSD2 induced apoptosis by inactivation of survival signaling component Akt, and activation of the caspase cascade (mitochondrial pathway) in HeLa cells. The results indicated a potential tumor suppressive function of SUSD2. Conversely, Watson et al (5) reported that SUSD2 was expressed at high levels in human breast cancer. The study suggested that SUSD2 may enhance the invasive ability of breast cancer cells and potentially contribute to a immune evasion mechanism by inducing apoptosis of Jurkat T cells. However, to the best of our knowledge, SUSD2 expression status and its correlation with the clinicopathological features of NSCLC have not previously been investigated.

In the present study, SUSD2 messenger RNA (mRNA) and protein expression were evaluated in NSCLC and normal lung tissues by SYBR Green quantitative polymerase chain reaction 
(qPCR) and western blot analysis of samples obtained from 24 patients with NSCLC. Immunohistochemical (IHC) analysis for SUSD2 was performed on an NSCLC tissue microarray (TMA) obtained from 192 patients with NSCLC. In addition, the potential associations between SUSD2 protein expression and the clinicopathological characteristics of NSCLC were analyzed.

\section{Materials and methods}

Patients and tissue samples. In the present study, 24 paired NSCLC and adjacent normal lung tissue samples were collected from patients between June 2012 and May 2014 for reverse transcription-qPCR (RT-qPCR) and western blot analysis. All samples were collected by resection from patients with lung cancer at the Department of Thoracic Surgery, Nanfang Hospital of Southern Medical University (Guangzhou, China). The samples selected were from patients with pathologically diagnosed cases of NSCLC, having received no chemotherapy or radiotherapy prior to surgery. NSCLC tissue microarrays, including 160 formalin-fixed, paraffin-embedded NSCLC tissues and 32 normal lung tissues, were purchased from Alena Biotech (Xi'an, China). The ages of the 192 patients with NSCLC ranged from 14-81 years. Clinicopathological features of the patients, including gender, age at diagnosis, histological grade, clinical stage, pathology type and pTNM status, are listed in Table I. All cases were histologically classified as NSCLC and the cancer stages were classified according to the American Joint Committee on Cancer criteria (8). The present study was approved by the Ethics Committee of the Nanfang Hospital and conducted with written informed consent from all patients included.

$R N A$ isolation and $R T-q P C R$ analysis. Total RNAs were isolated from 24 pairs of fresh NSCLC and adjacent normal lung tissues using RNAiso Plus reagent (Takara Biotechnology, Co., Ltd, Dalian, China). RNA concentration and optical density 260/280 were assessed by using a BioPhotometer Plus spectrophotometer (Eppendorf, Hamburg, Germany). First-strand complementary DNA (cDNA) was prepared from total RNA using the PrimeScript ${ }^{\circledR}$ RT reagent kit (Takara Biotechnology, Co., Ltd, Dalian, China). The RT reaction was performed under the following conditions: $37^{\circ} \mathrm{C}$ for $15 \mathrm{~min}$ and $85^{\circ} \mathrm{C}$ for $5 \mathrm{sec}$, followed by $42^{\circ} \mathrm{C}$ for $2 \mathrm{~min}$. The $20 \mu \mathrm{l}$ PCR reaction mixture included $50 \mathrm{ng}$ cDNA template, $0.4 \mu \mathrm{M}$ each of the forward and reverse primers, $10 \mu 1$ 2X SYBR Premix Ex Taq ${ }^{\mathrm{TM}}$ II, $0.4 \mu \mathrm{l}$ ROX Reference Dye II (Takara Biotechnology, Co., Ltd) and double distilled $\mathrm{H}_{2} \mathrm{O}$. The primers for the SUSD2 gene were: Forward, 5'-CTCCAATGACTGCCGCAACTA-3 and reverse, 5'-GAACATTCCTTTCAGGTCCATCC-3'. The primers for the $\beta$-actin gene were: Forward, 5'-AGCCTCGCCTTTGCCGA-3, and reverse, 5'-CTGGTGCCTGGGGCG-3' (9). The qPCR reaction was performed in an ABI 7500 real-time PCR amplifier (Applied Biosystems Life Technologies, Foster City, CA, USA) with an initial denaturing temperature of $95^{\circ} \mathrm{C}$ for $30 \mathrm{sec}$, followed by 40 cycles of $95^{\circ} \mathrm{C}$ for $5 \mathrm{sec}$ and $60^{\circ} \mathrm{C}$ for $30 \mathrm{sec}$. The $\mathrm{Ct}$ values were acquired using the 7500 system SDS software, version 2.0.1 (Applied Biosystems Life Technologies) with manual thresholds. $\beta$-actin was used
Table I. AUC for each clinicopathological feature.

\begin{tabular}{lcc}
\hline Characteristic & AUC $(95 \% \mathrm{CI})$ & P-value \\
\hline Gender & $0.540(0.438-0.643)$ & 0.441 \\
Age & $0.539(0.444-0.633)$ & 0.405 \\
Histological grade & $0.799(0.715-0.883)$ & 0.000 \\
Clinical stage & $0.548(0.435-0.661)$ & 0.427 \\
Pathology type & $0.593(0.505-0.681)$ & 0.043 \\
pT status & $0.533(0.421-0.645)$ & 0.581 \\
pN status & $0.601(0.499-0.703)$ & 0.042 \\
pM status & $0.740(0.572-0.908)$ & 0.245 \\
\hline
\end{tabular}

AUC, area under receiver operating characteristic curve; CI, confidence interval.

as an internal control gene to normalize PCR to the quantity of RNA added to the RT reactions (10). Fold-changes between NSCLC and normal tissue pairs were analyzed by calculating the $2^{-\Delta \Delta C t}$ values (11). Each PCR reaction was repeated in triplicate for stable results.

Western blot analysis. Total proteins from 24 pairs of fresh NSCLC and adjacent normal tissues were extracted using radioimmunoprecipitation assay buffer containing $1 \mathrm{mM}$ phenylmethylsulfonyl fluoride (Beyotime Institute of Biotechnology, Haimen, China). Western blotting was performed according to the method described by Lee et al (11). Proteins were separated on NuPAGE 4-12\% bis-Tris polyacrylamide gels (Invitrogen Life Technologies, Carlsbad, CA, USA) and then electrophoretically transferred to polyvinylidene fluoride membranes (EMD Millipore, Billerica, MA, USA) by a Transblot SD Cell semi-dry transfer machine (Bio-Rad Laboratories, Inc., Hercules, CA, USA). The membranes were then incubated for $1 \mathrm{~h}$ at room temperature, with a 1:1,000 dilution of rabbit polyclonal anti-SUSD2 antibody (Sigma-Aldrich, St. Louis, MO, USA). Subsequently, horseradish peroxidase (HRP)-conjugated goat anti-rabbit immunoglobulin G (Multisciences, Hangzhou, China) was applied at a dilution of 1:2,000 for $2 \mathrm{~h}$ at room temperature. Anti- $\beta$-actin antibody was used as a loading control and the signals were visualized using an enhanced chemiluminescence detection kit (GE Healthcare Life Sciences, Little Chalfont, UK).

IHC. NSCLC TMAs (LC1921; Alenabio, Xi'an, China) were constructed with 160 formalin-fixed, paraffin-embedded NSCLC tissues and 32 normal lung tissues. The TMA slides were deparaffinized in xylene and rehydrated in graded ethanol. The sections were subsequently immersed in $3 \%$ hydrogen peroxide solution for 10 min to block endogenous peroxidase activity. Antigen retrieval was performed by microwave heating with sodium citrate buffer $\left(\mathrm{pH} \mathrm{6.0)}\right.$ at $100^{\circ} \mathrm{C}$ for $20 \mathrm{~min}$. The TMA slides were blocked with 5\% normal goat serum at room temperature for $1 \mathrm{~h}$, followed by incubation with polyclonal rabbit anti-SUSD2 antibody (1:200; Sigma-Aldrich) at $4^{\circ} \mathrm{C}$ overnight, and polymer peroxidase-labeled anti-rabbit secondary antibody (ZSGB-Bio, Beijing, China) at room temperature for $45 \mathrm{~min}$ (12). The TMA slides were stained 
A

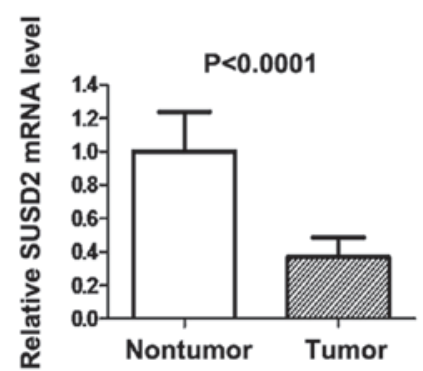

C

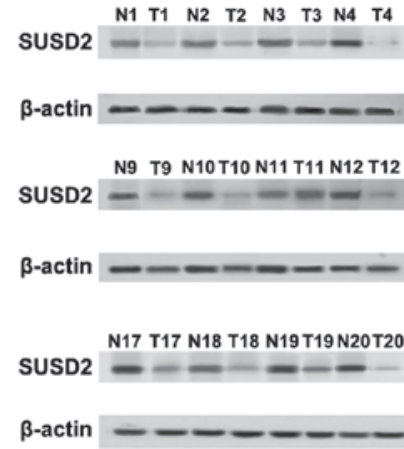

B

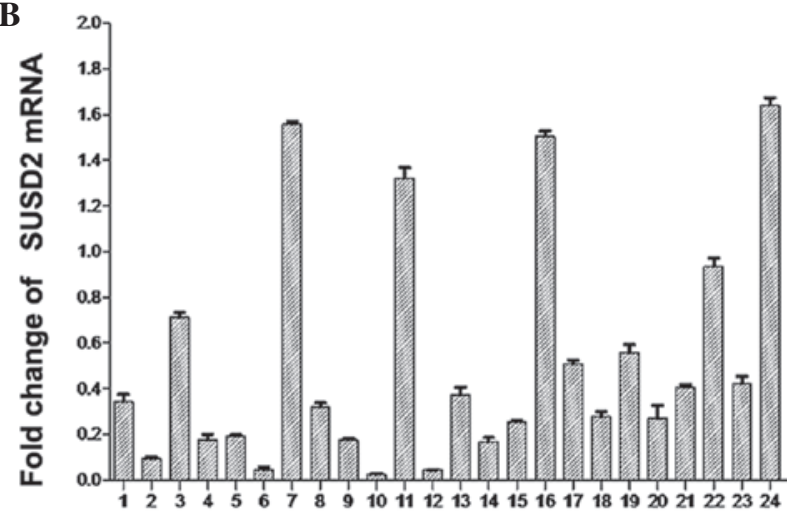

$\begin{array}{lllllllll}\text { N5 } & \text { T5 } & \text { N6 } & \text { T6 } & \text { N7 } & \text { T7 } & \text { N8 } & \text { T8 }\end{array}$

D
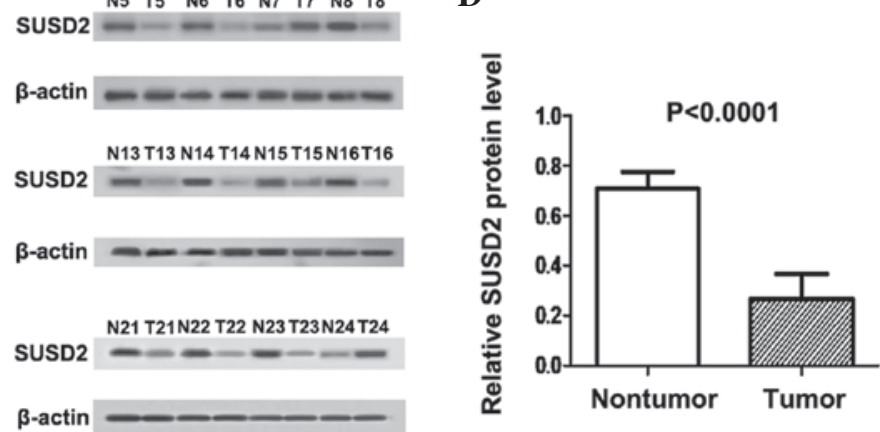

Figure 1. RT-qPCR and western blot analysis of SUSD2 expression in paired NSCLC and adjacent normal lung tissues. (A) Significant differences in SUSD2 mRNA expression between the NSCLC and adjacent normal lung tissues $(\mathrm{P}<0.0001)$. (B) Fold-changes $\left(2^{-\Delta \Delta \mathrm{Ct}}\right.$ values) detected by RT-qPCR revealed reduced expression of SUSD2 mRNA in the majority of NSCLC cases (20/24), when compared with that of paired normal lung tissues. Expression levels were normalized to $\beta$-actin. (C) Western blotting indicated downregulation of SUSD2 protein expression in NSCLC tissues (21/24), compared with that of adjacent normal lung tissues. $\beta$-actin was used as an internal control. (D) Significant differences in SUSD2 protein expression between the NSCLC and adjacent normal lung tissues $(\mathrm{P}<0.0001)$. RT-qPCR, reverse transcription-quantitative polymerase chain reaction; SUSD2, sushi domain containing 2; NSCLC, non-small cell lung cancer; mRNA, messenger RNA; T, NSCLC; N, normal.

using the diaminobenzidene HRP Color Development kit (Beyotime Institute of Biotechnology), and then counterstained with hematoxylin (Chemical reagent factory, Guangzhou, China). A negative control was performed without the primary antibodies, and a known IHC-positive slide (Alenabio) was used as a positive control $(13,14)$.

IHC evaluation. The intensity of SUSD2 protein staining of the 192 lung samples was scored using a semiquantitative scale as previously described (15). Briefly, with regard to distribution of SUSD2 protein, the scores were expressed as a percentage of positive tumor cells over the total tumor cells, in $5 \%$ increments $(0-100 \%)$. Two investigators graded the immunohistochemical expression of SUSD2 and the scores were accepted if the scores determined by the two investigators agreed. Otherwise, values were re-estimated until a consensus was reached.

Selection of cut-off score and statistical analysis. Receiver operating characteristic (ROC) curve analysis was used to determine an optimal cut-off score for the positive expression of SUSD2 using the 0,1-criterion (15). The ROC curves were generated by plotting the sensitivity and specificity for each outcome at various SUSD2 scores. The corresponding area under the ROC curve (AUC) and P-value were analyzed using SPSS 18.0 software (SPSS, Inc., Chicago, IL, USA). The score closest to the point $(0.0,1.0)$ on the curve, with maximum sensitivity and specificity, was selected as the cut-off value (15). Dichotomization of the clinicopathological features for ROC analysis were as follow: Gender (male vs. female), age ( $\leq 55$ vs. $>55$ years), histology grade (I-II vs. III), clinical stage (I-II vs. III-IV), pathology type (adenocarcinoma vs. squamous cell carcinoma), pT stage (T1-T2 vs. T3-T4), pN stage (N0 vs. N1-N2) and pM stage (M0 vs. M1). Statistical analyses were performed using SPSS 18.0 software. The association of SUSD2 expression with the clinicopathological features of NSCLC patients was analyzed by the $\chi^{2}$ test. $\Delta \mathrm{Ct}$ and grey value of lung cancer tissue samples and nomal tissues was tested by paired-sample Student's t-test. $\mathrm{P}<0.05$ was considered to indicate a statistically significant difference (two-tailed test) (16).

\section{Results}

SUSD2 $m R N A$ and protein expression levels are downregulated in NSCLC tissues. As shown in Fig. 1A, SUSD2 mRNA expression levels in cancer tissues were significantly lower than those in adjacent normal tissues $(\mathrm{P}<0.0001)$. RT-qPCR analysis indicated that SUSD2 mRNA expression was downregulated in NSCLC tissues compared with adjacent normal lung tissues in 20/24 sample pairs (Fig. 1B). Western blot analysis demonstrated that expression of the SUSD2 protein was also downregulated in 21/24 NSCLC tissues compared with their adjacent normal counterparts (Fig. 1C). The difference 
A

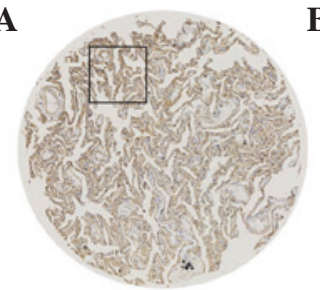

F

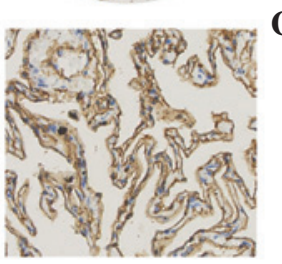

B
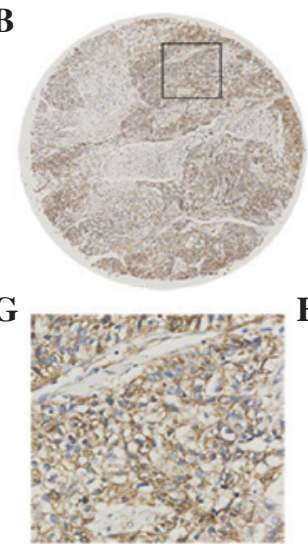

C

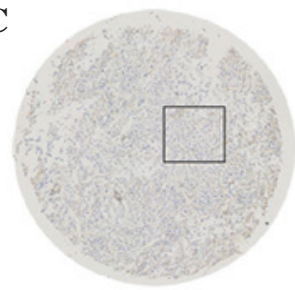

$\mathbf{H}$

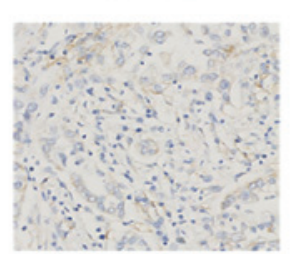

D

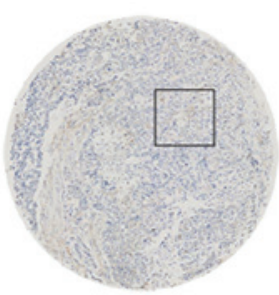

I

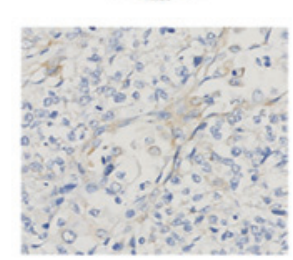

$\mathbf{E}$

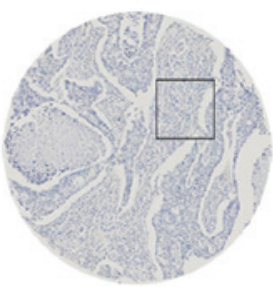

$\mathbf{J}$

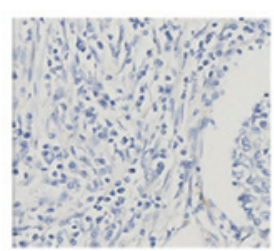

Figure 2. Expression of SUSD2 protein in normal lung and NSCLC tissues by immunohistochemical analysis of the tissue microarray. (A) Positive expression of SUSD2 in normal lung tissue, in which $90 \%$ of the lung cells demonstrated marked membrane staining of SUSD2, while lesser staining was also observed in the cytoplasm (magnification, x100). (B) Positive expression of SUSD2 in NSCLC (case 13), in which $>90 \%$ of tumor cells exhibited positive staining (magnification, x100). (C) Negative expression of SUSD2 in NSCLC (case 168), with <35\% positive tumor cells (magnification, $x 100)$. (D) Negative expression of SUSD2 in NSCLC (case 74), with <20\% positive tumor cells (magnification, x100). (E) Negative expression of SUSD2 in NSCLC (case 161), with <5\% positive tumor cells (magnification, x100). (F-J) Higher magnification (x400) of the black squares outlined in A-E, respectively. SUSD2, sushi domain containing 2; NSCLC, non-small cell lung cancer.

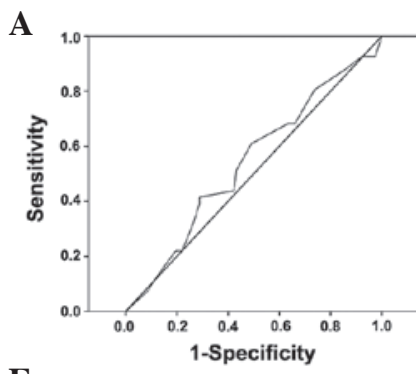

$\mathbf{E}$

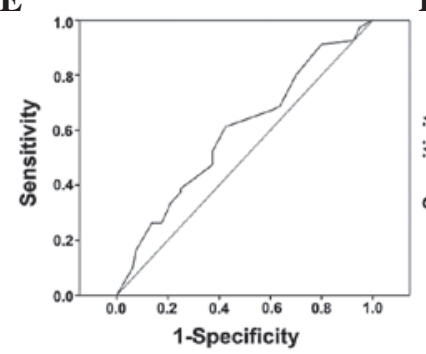

B

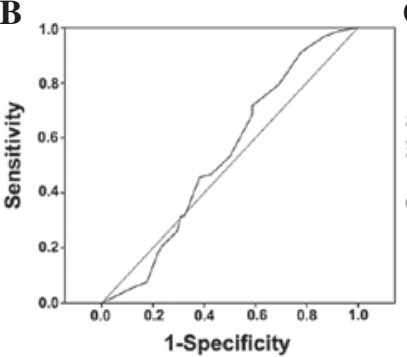

F

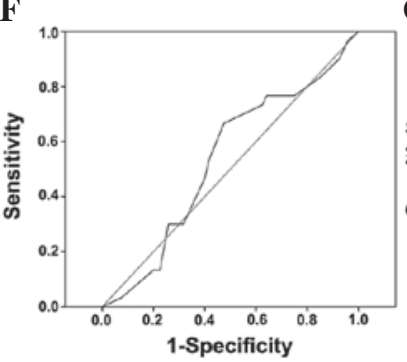

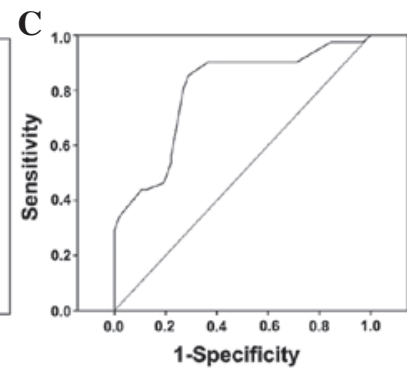

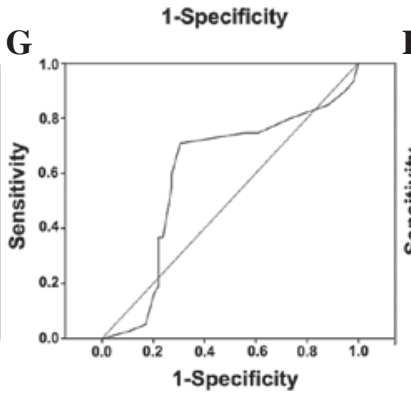

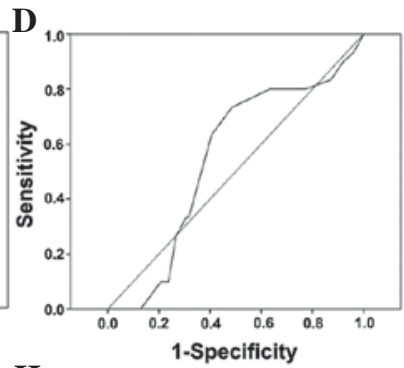

H

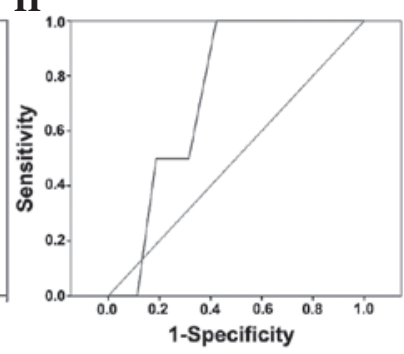

Figure 3. Selection of the optimum cut-off score for positive expression of sushi domain containing 2 by ROC curve analysis. Various ROC curves were plotted by sensitivity and specificity for each outcome: (A) Gender, (B) age, (C) histological grade, (D) clinical stage, (E) pathology type, (F) pT status, (G) pN status, (H) pM status. ROC, receiver operating characteristic.

in SUSD2 protein expression between the two groups was statistically significant, as indicated by paired t-test $(\mathrm{P}<0.0001$; Fig. 1D).

Positive rate of SUSD2 expression is downregulated in NSCLC tissues. The subcellular localization and expression of SUSD2 protein were observed and scored by IHC analysis of the TMA, which included 160 NSCLC and 32 normal lung tissues. The variation in SUSD2 expression levels observed in the NSCLC samples varied between 0 and $100 \%$. As indicated in Fig. 2, positive SUSD2 immunohistochemical staining was predominantly observed on the cell membrane, while lower levels of staining were also found in the cytoplasm.

ROC curves for the clinicopathological characteristics at various SUSD2 scores were plotted (Fig. 3). The optimal cut-off score for SUSD2 was determined by the ROC curve for histological grade, which was closest to the point $(0.0$, 1.0). According to the cut-off score, tissues were defined as SUSD2 positive when the SUSD2 expression percentage was $>47.5 \%$. Based on the SUSD2 scores for each sample and the cut-off score determined by ROC curve analysis, the positive rate of SUSD2 in cancer and normal tissues were 55\% (88/160) and $100 \%$ (32/32), respectively, which indicated a statistically significant difference $\left(\chi^{2}=23.040 ; \mathrm{P}<0.000\right)$.

Correlation between SUSD2 expression and clinicopathological characteristics of patients with NSCLC. In order to reveal the clinical significance of SUSD2 protein expression levels in the NSCLC tissues, the correlation between SUSD2 protein expression in the cancer tissues and various 
Table II. Association between SUSD2 expression and clinicopathological features of patients with non-small cell lung cancer.

\begin{tabular}{|c|c|c|c|c|}
\hline \multirow[b]{2}{*}{ Clinicopathological feature } & \multicolumn{2}{|c|}{ SUSD2 staining } & \multirow[b]{2}{*}{ Total, n } & \multirow[b]{2}{*}{ P-value ${ }^{a}$} \\
\hline & Negative, n (\%) & Positive, n (\%) & & \\
\hline Gender & & & & 0.375 \\
\hline Male & $51(43.2)$ & $67(56.8)$ & 118 & \\
\hline Female & $21(51.2)$ & $20(48.8)$ & 41 & \\
\hline Age at surgery, years & & & & 0.607 \\
\hline$\leq 55$ & $29(42.6)$ & $39(57.4)$ & 68 & \\
\hline$>55$ & $43(46.7)$ & $49(53.3)$ & 92 & \\
\hline Histological grade & & & & 0.000 \\
\hline 1 & $1(6.7)$ & $14(93.3)$ & 15 & \\
\hline 2 & $26(31.7)$ & $56(68.3)$ & 82 & \\
\hline 3 & $35(85.4)$ & $6(14.6)$ & 41 & \\
\hline Clinical stage & & & & 0.013 \\
\hline $\mathrm{I}$ & $14(29.2)$ & $34(70.8)$ & 48 & \\
\hline II & $27(50.9)$ & $26(49.1)$ & 53 & \\
\hline III & $17(60.7)$ & $11(39.3)$ & 28 & \\
\hline IV & $2(100)$ & $0(0.0)$ & 2 & \\
\hline Pathology & & & & 0.057 \\
\hline Adenocarcinoma & $30(37.5)$ & $50(62.5)$ & 80 & \\
\hline Squamous cell carcinoma & $42(52.5)$ & $38(47.5)$ & 80 & \\
\hline pT status & & & & 0.028 \\
\hline $\mathrm{T} 1$ & $1(7.7)$ & $12(92.3)$ & 13 & \\
\hline $\mathrm{T} 2$ & $49(45.8)$ & $58(54.2)$ & 107 & \\
\hline $\mathrm{T} 3$ & $15(51.7)$ & $14(48.3)$ & 29 & \\
\hline $\mathrm{T} 4$ & $1(100)$ & $0(0.0)$ & 1 & \\
\hline Lymph node metastasis & & & & 0.002 \\
\hline No & $16(27.1)$ & 43 (72.9) & 59 & \\
\hline N1 & $40(60.6)$ & $26(39.4)$ & 66 & \\
\hline $\mathrm{N} 2$ & $6(50)$ & $6(50)$ & 12 & \\
\hline N3 & $1(100)$ & $0(0.0)$ & 1 & \\
\hline Distant metastasis & & & & 0.121 \\
\hline No & $67(45)$ & $82(55)$ & 149 & \\
\hline Yes & $2(100)$ & $0(0.0)$ & 2 & \\
\hline
\end{tabular}

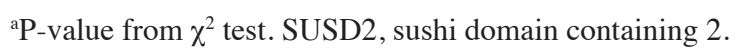

clinicopathological parameters was analyzed (Table II). The result indicated that reduced expression of SUSD2 in NSCLC patients was correlated with poor histological grade $\left(\chi^{2}=41.764 ; \mathrm{P}<0.000\right)$, advanced clinical stage $\left(\chi^{2}=10.790\right.$; $\mathrm{P}=0.013)$, higher $\mathrm{pT}\left(\chi^{2}=9.070 ; \mathrm{P}=0.028\right)$ and positive regional lymph node metastasis $\left(\chi^{2}=15.399 ; \mathrm{P}=0.002\right)$. No correlation was observed between SUSD2 expression and other features analyzed, including gender, age, pathology type or pM status $(\mathrm{P}>0.05)$.

\section{Discussion}

Worldwide, lung cancer has demonstrated a significant rising trend in terms of morbidity and mortality in recent years, and has become a significant hazard to human health and life (15).
Due to the difficulties associated with the early detection of lung cancer, the majority of patients with NSCLC present with advanced stage cancer at diagnosis (17). Conventional platinum-based chemotherapy and radiation have low efficacy against this malignancy due to its frequent recurrence and distant metastasis, and the overall 5-year survival rate of patients with NSCLC remains at $<15 \%(18,19)$. Therefore, considerable efforts are required to explore novel biomarkers and improve the early diagnosis and targeted therapeutic interventions of NSCLC.

SUSD2 is a transmembrane protein that is widely expressed in human tissues. Two previous studies $(6,7)$ have been published describing the mouse homolog SUSD2 as a potential tumor suppressor. By contrast, Watson et al (5) reported that SUSD2 was overexpressed in breast cancer, and 
indicated that SUSD2 enhanced the invasion of breast cancer cells. Accelerated tumor formation and decreased survival rates in mice with tumors expressing SUSD2 were observed in a syngeneic mouse model (5). However, the underlying physiopathological role of SUSD2 in human NSCLC has remained to be elucidated. In the present study, the expression levels of SUSD2 mRNA and protein were analyzed by RT-qPCR and western blot analysis in paired NSCLC and adjacent normal lung tissue samples. The results revealed that the expression of SUSD2 mRNA and protein was decreased in the majority of cancer tissues, when compared with their paired adjacent normal lung tissues $(\mathrm{P}<0.001)$. Furthermore, IHC analysis of SUSD2 on a TMA, including 160 NSCLC and 32 normal lung tissues, was also conducted on various pathological types, histological grades and clinical stages of NSCLC. In the present study, reduced expression of SUSD2 was frequently observed in NSCLC tissues, while all normal lung tissues exhibited marked staining of SUSD2. This was in accordance with the results of the RT-qPCR and western blot analyses.

In order to reveal the prognostic value of SUSD2 expression in NSCLC tissues, the correlation between SUSD2 protein levels and clinicopathological characteristics was analyzed. The results indicated that reduced expression of SUSD2 was correlated with poor histological grade, advanced clinical stage, higher $\mathrm{T}$ status and higher $\mathrm{N}$ status. These results indicated that low expression levels of SUSD2 were correlated with the aggressive behavior of NSCLC, which suggested the potential value of SUSD2 protein expression level evaluation in the determination of NSCLC progression. However, to fully elucidate the specific function of SUSD2 in NSCLC, further investigations with larger cohorts are required.

In conclusion, the current study demonstrated that SUSD2 was downregulated in human NSCLC tissues and was closely correlated with poor differentiation and aggressive behavior of NSCLC, indicating a potential role of the SUSD2 protein in the pathogenesis of NSCLC.

\section{Acknowledgements}

The present study was supported by grants from the Guangdong Supporting Grant for Outstanding Talent (no. C1030925) and the Natural Science Foundation of Guangdong Province (no. S2013010016631).

\section{References}

1. Brody H: Lung cancer. Nature 513: S1, 2014.

2. Salgia R: Prognostic significance of angiogenesis and angiogenic growth factors in nonsmall cell lung cancer. Cancer 117 3889-3899, 2011.
3. Siegel R, Naishadham D and Jemal A: Cancer statistics for Hispanics/Latinos, 2012. CA Cancer J Clin 62: 283-298, 2012.

4. Govindan R, Page N, Morgensztern D, Read W, Tierney R, Vlahiotis A, Spitznagel EL and Piccirillo J: Changing epidemiology of small-cell lung cancer in the United States over the last 30 years: Analysis of the surveillance, epidemiologic and end results database. J Clin Oncol 24: 4539-4544, 2006

5. Watson AP, Evans RL and Egland KA: Multiple functions of sushi domain containing 2 (SUSD2) in breast tumorigenesis. Mol Cancer Res 11: 74-85, 2013.

6. Sugahara T, Yamashita Y, Shinomi M, Yamanoha B, Iseki H, Takeda A, Okazaki Y, Hayashizaki Y, Kawai K, Suemizu H and Andoh T: Isolation of a novel mouse gene, mSVS-1/SUSD2, reversing tumorigenic phenotypes of cancer cells in vitro. Cancer Sci 98: 900-908, 2007.

7. Sugahara T, Yamashita Y, Shinomi M, Isobe Y, Yamanoha B, Iseki H, Takeda A, Okazaki Y, Kawai K, Suemizu H and Andoh T: von Willebrand factor type D domain mutant of SVS-1/SUSD2, vWd(m), induces apoptosis in HeLa cells. Cancer Sci 98: 909-915, 2007.

8. Chheang S and Brown K: Lung Cancer staging: Clinical and radiologic perspectives. Semin Intervent Radiol 30: 99-113, 2013.

9. Kreuzer KA, Lass U, Landt O, Nitsche A, Laser J, Ellerbrok H, Pauli G, Huhn D and Schmidt CA: Highly sensitive and specific fluorescence reverse transcription-PCR assay for the pseudogene-free detection of beta-actin transcripts as quantitative reference. Clin Chem 45: 297-300, 1999.

10. Baine MJ, Mallya K and Batra SK: Quantitative real-time PCR expression analysis of peripheral blood mononuclear cells in pancreatic cancer patients. Methods Mol Biol 980: 157-173, 2013.

11. Livak KJ and Schmittgen TD: Analysis of relative gene expression data using real-time quantitative PCR and the 2 (-Delta Delta C(T)) method. Methods 25: 402-408, 2001.

12. Lee YJ, Kim DH, Lee SH, Kim DW, Nam HS and Cho MK: Expression of the c-Met proteins in malignant skin cancers. Ann Dermatol 23: 33-38, 2011

13. Shi M, Yu DH, Chen Y, Zhao CY, Zhang J, Liu QH, Ni CR and $\mathrm{Zhu} \mathrm{MH}$ : Expression of fibroblast activation protein in human pancreatic adenocarcinoma and its clinicopathological significance. World J Gastroenterol 18: 840-846, 2012.

14. Shan LH, Sun WG, Han W, Qi L, Yang C, Chai CC, Yao K, Zhou QF, Wu HM, Wang LF and Liu JR: Roles of fibroblasts from the interface zone in invasion, migration, proliferation and apoptosis of gastric adenocarcinoma. J Clin Pathol 65: 888-895, 2012.

15. Shi R, Zhao Z, Zhou H, Wei M, Ma WL, Zhou JY and Tan WL: Reduced expression of PinX1 correlates to progressive features in patients with prostate cancer. Cancer Cell Int 14: 46, 2014.

16. Wang $\mathrm{G}$, Wu J and Song H: LRIG2 expression and prognosis in non-small cell lung cancer. Oncol Lett 8: 667-672, 2014.

17. Saintigny P and Burger JA: Recent advances in non-small cell lung cancer biology and clinical management. Discov Med 13: 287-297, 2012.

18. Konishi J, Yamazaki K, Azuma M, Kinoshita I, Dosaka-Akita H and Nishimura M: B7-H1 expression on non-small cell lung cancer cells and its relationship with tumor-infiltrating lymphocytes and their PD-1 expression. Clin Clin Cancer Res 10: 5094-5100, 2004.

19. Molina JR, Yang P, Cassivi SD, Schild SE and Adjei AA: Non-small cell lung cancer: Epidemiology, risk factors, treatment and survivorship. Mayo Clin Proc 83: 584-594, 2008. 